\title{
Juntas de Usuarios de los Servicios de Salud en el Perú: conocimientos, percepciones y prácticas sobre la protección de sus derechos
}

\section{Health Services Users Boards in Peru: knowledge, perceptions and practices on the protection of their rights}

\author{
María Jeri-De-Pinho ${ }^{1, a}$ \\ ${ }^{1}$ Universidad Nacional Mayor de San Marcos. Lima, Perú. \\ a Licenciada en Trabajo Social.
}

Tesis presentada para obtener el grado académico de Magister en Política Social, Gestión de Proyectos y Programas Sociales de la Facultad de Ciencias Sociales de la Universidad Nacional Mayor de San Marcos

\begin{abstract}
Correspondencia:
María Stephanie Jeri De Pinho

stephaniejeridepinho@gmail.com

Teléfono: 969793686

Recibido: 23 de agosto 2017

Aceptado: 26 de febrero 2018

Conflictos de interés: Ninguno.

Fuentes de financiamiento:

Autofinanciado.
\end{abstract}

Citar como: Jeri-De-Pinho M. Juntas de Usuarios de los Servicios de Salud en el Perú: conocimientos, percepciones y prácticas sobre la protección de sus derechos. An Fac med. 2018;79(1):17-21 DOI: http://dx.doi.org/10.15381/anales. v79i1.14587

\section{Resumen}

Introducción. La participación social y el empoderamiento de los ciudadanos constituyen una función esencial de la salud pública, necesaria para la acción colectiva de protección, promoción, y mejora de la salud. La Superintendencia Nacional de Salud (SUSALUD) es una institución que protege los derechos en salud, empoderando al ciudadano en el centro del sistema de salud nacional, promoviendo la responsabilidad compartida mediante las Juntas de Usuarios de los Servicios de Salud (JUS). El objetivo del estudio fue determinar los conocimientos, percepciones y prácticas de los miembros de las JUS sobre el marco normativo y funciones de SUSALUD y las JUS, en el periodo 2015-2016. Métodos. Estudio observacional, descriptivo en siete regiones del Perú con la participación de 67 miembros de las JUS. Se aplicó una encuesta de medición de conocimientos, percepciones, y prácticas. Resultados. Los 67 miembros de las JUS fueron mayores de 18 años, $56,7 \%$ con estudios superiores, $58,2 \%$ dentro de la población económicamente activa, y $70,1 \%$ participó previamente de una organización. Más del $60 \%$ de encuestados conocian el marco normativo y funciones de SUSALUD y las JUS. En lo referente a los conocimientos, percepciones y prácticas, existe una predisposición a la difusión y promoción de la participación ciudadana de los usuarios de servicios de salud. Conclusiones. Los conocimientos, percepciones y prácticas de los miembros de las JUS de siete regiones del Perú son adecuados para la estrategia de materializar la participación ciudadana en la gestión institucional para la contribución a la protección y promoción de la salud.

Palabras clave: Participación Social; Salud Pública; Protección; Promoción de la Salud; Conocimiento (fuente: DeCS BIREME).

\section{Abstract}

Introduction. Social participation and empowerment of citizens is an essential public health function, necessary for collective action of protection, promotion and improvement of health. The National Superintendence of Health (SUSALUD) is an institution that protects health rights by empowering citizens at the center of the national health system, promoting shared responsibility through the Health Services Users Boards (JUS). The objetive of the present study was determine the knowledge, perceptions and practices of the members of the JUS on the normative framework and functions of SUSALUD and the JUS, in the period 2015-2016. Methods. Observational, descriptive study in seven regions of Peru with the participation of 67 members of the JUS. A survey was applied to measure knowledge, perceptions, and practices. Results. The 67 members of the JUS were older than 18 years, 56.7\% with higher education, $58.2 \%$ within the economically active population, and $70.1 \%$ previously participated in an organization. More than $60 \%$ of respondents knew the normative framework and functions of SUSALUD and the JUS. With regard to knowledge, perceptions and practices, there is a predisposition to the dissemination and promotion of citizen participation of users of health services. Conclusions. The knowledge, perceptions and practices of JUS members from seven regions of Peru are adequate for the strategy to materialize citizen participation in institutional management to contribute to the protection and Health promotion.

Keywords: Social Participation ; Public Health ; Protection; Health Promotion; Knowledge (source: MeSH NLM). 


\section{INTRODUCCIÓN}

Frente a los cambios estructurales y financieros adoptados en los procesos de reforma en salud en las Américas, se ha propuesto a la salud pública como responsabilidad social de los gobiernos ${ }^{(1)}$. Para ello, la OPS ha definido funciones esenciales de salud pública, medibles para su cumplimiento tanto por el Estado como por la sociedad civil en tanto se desarrolle una acción colectiva para la protección, promoción, y mejoramiento de salud de las personas dado que constituyen elementos esenciales para garantizar el bienestar humano y el desarrollo socioeconómico de las naciones ${ }^{(1,2,3)}$; así, se definió a la participación social y empoderamiento de los ciudadanos en salud como una función esencial de salud pública, recomendando a los gobiernos el fortalecimiento de la construcción de alianzas intersectoriales con la sociedad civil que permitan utilizar el capital humano y los recursos materiales disponibles para mejorar el estado de salud de la población así como promover ambientes que favorezcan la vida saludable ${ }^{(1)}$.

La Superintendencia Nacional de Salud (SUSALUD) es un organismo adscrito al Ministerio de Salud, que cuenta con autonomía técnica, funcional, administrativa, y financiera, con la finalidad de promover, proteger y defender los derechos de las personas al acceso a los servicios de salud, supervisando que las prestaciones sean otorgadas con calidad, oportunidad, disponibilidad y aceptabilidad, con independencia de quien las financie ${ }^{(4)}$; así, es una institución que protege los derechos en salud a nivel nacional como necesidad de interés de la población de ejercer sus derechos en materia de salud, considerada como una política pública y social en concordancia con los objetivos de la Reforma del Sector Salud en nuestro país ${ }^{(5)}$.

La protección de los derechos en salud se encuentra relacionada con el derecho a la participación de los usuarios en la gestión de los servicios de salud, pasando de una relación de clientes a sujetos con capacidades, información y organización para el ejercicio de sus derechos en salud ${ }^{(6)}$. En ese sentido, SUSALUD orienta sus acciones a empoderar al ciudadano en el centro del sistema de salud nacional, promoviendo la responsabilidad compartida a través de la retroalimentación mutua entre los sistemas institucionales y los sistemas ciudadanos, sin importar su condición de aseguramiento o las instituciones prestadoras de salud donde acude; para ello, SUSALUD cuenta con las Juntas de Usuarios de los Servicios de Salud (JUS), instancia que materializa la participación ciudadana en la gestión institucional, constituyéndose en un espacio de escucha y diálogo con los usuarios para contribuir con el mejoramiento de los fines referidos a la protección y promoción de la salud ${ }^{(7)}$. Las JUS cuentan con instrumentos normativos para regular sus actividades que son constantemente socializados a los miembros de dichas juntas; adicionalmente, cuentan con herramientas e instrumentos para su conformación y funcionamiento, facilitando la medición de sus resultados y avances. Dichas actividades son: emitir opinión sobre las consultas que SUSALUD plantea a la Junta; difundir entre la ciudadanía los proyectos de SUSALUD; emitir consultas, opiniones, así como informar sobre hechos que podrían implicar la vulneración de derechos en salud; proponer lineamentos de acción para contribuir con la promoción y protección del ejercicio de los derechos en salud de los usuarios de los servicios de salud; y promover la participación ciudadana de los servicios de salud así como el diálogo entre los usuarios y las entidades del sector salud(8).

En nuestro país son pocos los espacios de participación de los usuarios en materia de salud. Entre las experiencias más importantes tenemos las Comunidades Locales de Administración en Salud $(C L A S)^{(9)}$, el Consejo Nacional de Salud(10) y las JUS promovidas por SUSALUD ${ }^{(8)}$. Las dos primeras experiencias lograron impactar positivamente en las poblaciones más vulnerables y excluidas de nuestro país, y la tercera experiencia viene siendo analizada en diversas publicaciones ${ }^{(7,11,12)}$ incluida la que se expone en el presente artículo. Si bien SUSALUD realiza a través del INEI la encuesta anual de satisfacción de usuarios $^{(13)}$, es importante mencionar que no se dispone información de los propios miembros de las JUS referida a los conocimientos, percepciones y prácticas tanto del marco normativo y funciones de SUSALUD y las JUS así como las actividades que realizan en relación a la protección de sus derechos en salud, en la que intervienen factores culturales y generacionales, siendo finalmente el objetivo del presente estudio describir dicha información. Considerando que las JUS son actores fundamentales en la integración de SUSALUD con las instituciones prestadoras de salud, y con la participación ciudadana de los usuarios, es que se realizó la presente investigación en los miembros que componen dichas Juntas en siete regiones del Perú.

\section{MÉTODOS}

Se realizó un estudio observacional, descriptivo, en base a la información obtenida de fuentes secundarias de SUSALUD y por encuestas a los miembros de las Juntas de Usuarios de Servicios de Salud en siete regiones del país: Lima, Callao, Ayacucho, Arequipa, Cusco, Lambayeque, y Loreto. Se seleccionó a 67 miembros de las JUS mediante muestreo por conglomerados de una población de 68 miembros activos de dichas regiones ( 9 en Lima, 8 en Callao, 8 en Ayacucho, 10 en Arequipa, 8 en Cusco, 14 en Lambayeque y 11 en Loreto). Como parte del recojo de información a los miembros de las JUS, se determinó mediante encuestas y fuentes secundarias de información: sus características sociodemográficas; el tiempo de participación en las JUS; los conocimientos, percepciones y prácticas respecto al marco normativo y funciones de SUSALUD y las JUS; las actividades que realizan las JUS en relación a la protección de sus derechos en salud; y los planes de trabajo con niveles de ejecución por parte de las JUS. Se utilizó una encuesta personalizada como instrumento de recolección de datos; asimismo, se presentan los datos mediante estadística descriptiva.

\section{RESULTADOS}

Se encuestó a 67 miembros de Juntas de Usuarios de los Servicios de Salud de las regiones de Lima, Callao, Ayacucho, 
Arequipa, Cusco, Lambayeque, y Loreto, mayores de 18 años, siendo 55,2\% mujeres y $44,8 \%$ hombres. Según el grado de instrucción, el 56,7\% contaba con grado de instrucción superior, 29,9\% con grado de instrucción secundario, 10.4\% con grado de instrucción primario, y $3 \%$ con primaria incompleta. En relación a la ocupación, el 34,3\% manifestó ser trabajador dependiente, el 23,9\% trabajador independiente, el 13,4\% ama de casa, 4,5\% jubilados, 4,5\% estudiantes, 10,4\% otros y $9 \%$ no opinó. La participación de los miembros de las JUS en dichas Juntas fue de 43,3\% menor a 1 año, el 55,2\% de 2 a 3 años, y el 1,5\% de 3 años a más. Asimismo, el 70,1\% señaló que participó previamente de una organización.

Respecto al marco normativo y funciones de SUSALUD, los encuestados respondieron correctamente según la siguiente descripción: el 83,6\% señaló que es un organismo técnico especializado que promueve, protege y defiende los derechos en salud; $91 \%$ señaló que protege los derechos en salud de cada peruano; $62,7 \%$ señaló como norma de creación al Decreto Legislativo N¹158 que dispone medidas destinadas al fortalecimiento y cambio de denominación de SUSALUD; 79,1\% señaló como líneas de acción a la promoción y protección de los derechos en salud, prevención de la vulneración, restitución del derecho, investigación y desarrollo; 79,1\% señaló que la Intendencia de Promoción de Derechos de Salud es un órgano de línea responsable de establecer, implementar, conducir y promover las actividades orientadas a fortalecer el ejercicio de los derechos y deberes en salud; $71,6 \%$ señaló como pilares de acción de la Intendencia de Promoción de Derechos de Salud a la promoción de derechos y acciones de participación ciudadana.

Respecto al marco normativo y funciones de las Juntas de Usuarios de los Servicios de Salud (JUS), los encuestados respondieron correctamente según la siguiente descripción: El 79,1\% señaló que el marco normativo de las JUS es la Resolución de Superintendencia N060-2014-SUSALUD/S, Reglamento de la Junta de Usuarios de los Servicios de Salud; el 94\% señaló que se encuentra conformada por miembros agrupados de organizaciones de pacientes así como miembros no agrupados de los servicios de salud; el 55,2\%, señaló que existe un proceso electoral para las organizaciones y un proceso de selección aleatoria para personas no organizadas; $53,7 \%$ señaló que existe un reglamento del proceso electoral y selección aleatoria; el 71,6\% señaló al Plan Anual de Trabajo de la Junta de Usuarios de Servicios de Salud como instrumento utilizado para su funcionamiento; el 89,6\% manifestó tener como actividades difundir derechos $y$ deberes en salud, proponer lineamientos de acción, informando a SUSALUD sobre casos de posible vulneración de derechos así como emitir consultas; el 70,1\% señaló a la JUS como el modelo de participación ciudadana; el 95,5\% señaló que tuvieron reuniones de trabajo de las JUS para tomar acuerdos; el 94\% refirió planificar sus acciones; el 94\% señaló haber realizado acciones de capacitación a los miembros; el 88,1\% señaló que contaron con el apoyo de la Intendencia de Promoción de Derechos de Salud; y el 73,1\% señaló que realizaron reuniones con sus organizaciones.

Respecto a los conocimientos y prácticas de los miembros de las JUS encuestados, los resultados se muestran en la tabla 1.

Entre los años 2015 y 2016 las Juntas de Usuarios de los Servicios de Salud de Lima y Callao desarrollaron dos planes de trabajo. Para los casos de las JUS de Arequipa, Ayacucho, Cusco, Lambayeque, y
Loreto, desarrollaron un plan de trabajo durante el año 2016. En todos los casos se tuvo un nivel de ejecución de $100 \%$. Las actividades que desarrollaron en los planes de trabajo referidos a la protección de sus derechos en salud fueron: Sesiones de trabajo para emitir opinión sobre consultas de la Superintendencia; difusión de derechos y deberes en salud en los establecimientos de salud así como los proyectos de SUSALUD; capacitaciones a las JUS; propuestas de mejora respecto a los lineamientos de acción para la contribución de la promoción y protección del ejercicio de los derechos de salud; acuerdos de trabajo con los establecimientos de salud y sensibilización al personal de salud como parte de la promoción de la participación ciudadana en los derechos de salud.

\section{DISCUSIÓN}

La participación social en salud, definida como derecho y deber del individuo, establece una serie de mecanismos institucionales para la participación colectiva de los usuarios y el Estado en la protección y promoción de la salud, constituyendo una estrategia de empoderamiento ${ }^{(1,14)}$. La participación ciudadana es una necesidad para los Estados democráticos dado que permite retroalimentar sus acciones y mejorar la eficacia y eficiencia de sus intervenciones; precisamente, el papel del ciudadano es el de agente activo de las políticas sociales, interlocutor del

Tabla 1. Conocimientos, percepciones y prácticas de los miembros de las Juntas de Usuarios de los Servicios de Salud de SUSALUD en siete regiones del Perú $(n=67)$

\section{Sí}

No No opina

Conocimientos

¿Conoce sus derechos y deberes en salud?

Percepciones y prácticas

¿Difunde los derechos y deberes en salud?

¿Realiza acciones para la mejora de los servicios de salud en conjunto con los prestadores de servicios de salud?

¿Informa a la Superintendencia Nacional de Salud sobre casos de posible vulneración de derechos en salud?

¿Elabora propuestas de mejora de los servicios de salud? ¿Promueve la participación ciudadana de los usuarios de servicios de salud?

¿Realiza consultas a SUSALUD sobre las acciones que desarrolla? ¿Brinda opinión a SUSALUD sobre normativa existente?

\begin{tabular}{ccc}
$97 \%$ & - & $3 \%$ \\
$94 \%$ & $1,5 \%$ & $4,5 \%$ \\
$86,5 \%$ & $9 \%$ & $4,5 \%$ \\
$79,1 \%$ & $16,4 \%$ & $4,5 \%$ \\
$89,5 \%$ & $7.5 \%$ & $3 \%$ \\
$92,5 \%$ & $3 \%$ & $4,5 \%$ \\
$67,2 \%$ & $19,4 \%$ & $13,4 \%$ \\
$64,2 \%$ & $23,9 \%$ & $11,9 \%$ \\
\hline
\end{tabular}


Estado y agente del fortalecimiento de la sociedad civil de la cual forma parte, en concordancia con lo señalado por Serrano en el año 1998, sobre el rol del ciudadano en cuanto usuario de los servicios de salud y participe de las decisiones y gestiones asociadas a la implementación de programas sociales(15).

En nuestro país, en el año 2013, los esfuerzos para involucrar a los usuarios en el diseño, implementación y evaluación de la gestión pública en materia de salud tuvieron mayor impulso a partir del fortalecimiento de la Superintendencia Nacional de Salud, institución pública encargada de promover, proteger y defender los derechos de las personas al acceso a los servicios de salud(15); así, SUSALUD, promueve la participación, vigilancia ciudadana y mecanismos de rendición de cuentas a la comunidad mediante las Juntas de Usuarios de los Servicios de Salud (JUS), diseñando un conjunto de normas, instrumentos y procedimientos, así como asistencia técnica para garantizar la implementación de las actividades planificadas $s^{(4,7,16)}$, mostrándose como instrumento principal de sus acciones el plan de trabajo de las JUS, con una ejecución promedio de $100 \%$, coincidiendo con lo señalado por Tanaka en el año 2001, sobre la importancia de la participación para poder alcanzar con mayor eficacia el objetivo del desarrollo, así como una mayor eficiencia de las políticas sociales, desde el punto de vista del uso de los recursos $^{(22)}$.

De los 67 miembros de las JUS de las siete regiones encuestadas en el presente estudio, $56,7 \%$ de ellos contaba con estudios superiores y $29,9 \%$ con grado de instrucción secundario, sobre el cual destacamos el aporte tanto para sus funciones como para las actividades que desarrollan dentro de las Juntas, en relación a su nivel académico. Además, 58,2\% se encontraba dentro de la población económicamente activa sea como trabajador dependiente o independiente, hecho que mostraba el cumplimiento de funciones y actividades en las JUS fuera de sus horarios habituales de trabajo, evidenciando el compromiso e identificación con las Juntas, más aún que la pertenencia a las JUS no genera ninguna remuneración dado su carácter ad honorem. Resalta el hecho que el 70,1\% de los encuestados manifestó haber pertenecido previamente a una organización, lo que demuestra experiencia previa en diversas formas de participación ciudadana y capacidad de organización en beneficio de las funciones que desempeñan en las JUS.

Los resultados expuestos demuestran que la mayoría de miembros encuestados conocían el marco normativo y funciones tanto de SUSALUD como de las JUS. Así, los mayores valores de porcentaje respondidos para cada pregunta fueron precisamente para las respuestas acertadas. Como es lógico, corresponde a las autoridades de SUSALUD aclarar las confusiones que existan y dotar de mayor información a quienes en pequeño porcentaje no acertaron lo inquirido. En lo referente a los conocimientos, percepciones y prácticas de los miembros de las JUS de SUSALUD en siete regiones de nuestro país, según los hallazgos presentados existe una predisposición a la difusión y promoción de la participación ciudadana de los usuarios de servicios de salud, hecho que debería ser potenciado y difundido por parte de SUSALUD para que los usuarios de nuestro sistema de salud, en general, tomen interés en participar de dichas Juntas.

En un estudio en Colombia, referido a la percepción de la participación en salud, se determinó que el concepto de participación presentó diferentes sentidos según el actor analizado; así, mientras que los usuarios articulaban el concepto de aportar ideas y estar en espacios sociales, el personal de salud otorgaba el sentido de participación a las actividades comunitarias. Asimismo, los formuladores de políticas dotaban el sentido de evaluación y control de los servicios de salud por parte de la comunidad, para mejorar su calidad(14). Es preciso mencionar que en la experiencia colombiana se introdujo la participación social en la organización y control de las funciones del sistema en el año 1993 como parte de la reforma en salud que se dio en dicho país. Precisamente, para abordar los obstáculos y oportunidades para los actores de la participación social en salud, una investigación determinó que los usuarios identificaron como obstáculos a participar: la falta de conocimiento, apatía, y limitada apertura institucional; además, los usuarios tuvieron la percepción de que su participación podría conseguir cambios $^{(17)}$. Estos hallazgos difieren de los nuestros dado que en la presente investigación se evidenció el adecuado conocimiento de las funciones y actividades de las JUS así como la predisposición de los usuarios a la apertura institucional a través de SUSALUD.

En México, la estrategia de la participación social en salud denominada "aval ciudadano" fue implementada a partir del año 2001 en la que los usuarios sea de manera individual o en agrupaciones elegidas en base a reconocimiento o prestigio social, se incorporaron a vigilar el proceso de mejora de los servicios de salud, como garantes de un buen servicio en salud. Así, en un estudio realizado en ocho estados, se determinó que las personas que participaron del "aval ciudadano" provenían de diversos ámbitos: los de zona rural eran mujeres dedicadas a su hogar, con escolaridad promedio de educación básica, tenían experiencias previas de participación comunitaria y destacaban por haber colaborado en diferentes comités de salud; los de zonas urbanas eran personas con mayor escolaridad(18), tal como los hallazgos descritos en nuestro estudio. Asimismo, en el estudio en mención, se cataloga como colaboracionista el tipo de participación social de los usuarios mediante el "aval ciudadano", sugiriendo que para alcanzar un real empoderamiento ciudadano este debería ser voluntario y con experiencia previa de participación; siendo que en nuestros resultados los miembros de las JUS realizaron voluntariamente sus funciones, y la mayoría de ellos tuvieron experiencia previa en organizaciones, es que, efectivamente, se logra el empoderamiento deseado, situación que también concuerda con lo manifestado por Hevia en el año 2011, en relación con la actuación de un grupo de personas con personalidad jurídica o no en la que se comparten intereses y objetivos iguales o similares en relación al tema que los convoca, en búsqueda de beneficio tanto individual como colectivo ${ }^{(23)}$.

En Brasil, la Constitución Federal de 1988 creó el Sistema Único de Salud que 
establece la creación de Consejos de Salud en cada estado y municipio de ese país, la composición de dicho Consejo guarda paridad entre los usuarios de los servicios, las autoridades, los proveedores, y trabajadores del sector, siendo el objetivo principal de la presencia de los usuarios su participación en las deliberaciones sobre las políticas y los programas de salud así como ejercer el control social -incluidas las relacionadas al gasto- sobre los actos de las autoridades locales quienes deben implementar las directrices acordadas ${ }^{(19)}$. En nuestra realidad, la participación ciudadana en las políticas públicas en materia de salud tiene, más bien, un carácter consultivo como parte del Consejo Nacional de Salud del MIN$S A^{(10)}$. En Chile, a partir del año 1995 se implementaron las redes de participación social en el sector salud; dichas redes derivaron en Consejos Consultivos y de Desarrollo integrados por los usuarios, organizaciones de la comunidad local, y los trabajadores de salud, como instancias interlocutoras con las autoridades de los hospitales y de los consultorios de atención primaria ${ }^{(18)}$. Dichos Consejos, al igual que en Brasil, tienen capacidad negociadora con las entidades prestadoras de salud, a diferencia de nuestra realidad donde los fines de la participación social en salud tienen como fundamento la protección de los derechos y promoción de la salud. Así, la participación de la ciudadanía en el sistema de salud chileno tiene un límite en la toma de decisiones de políticas públicas ${ }^{(20)}$.

En conclusión, el diseño e implementación de la participación social en salud depende de los valores políticos y sociales en cada país, surgiendo como fenómeno integrador ${ }^{(18,21)}$. Los resultados de nuestro estudio permiten describir los conocimientos, percepciones y prácticas de aquellos ciudadanos que forman parte de las JUS como parte de la estrategia definida en nuestro país para materializar la participación ciudadana en la gestión institucional para la contribución del mejoramiento de los fines referidos a la protección y promoción de la salud(7). Así, el valor de nuestros hallazgos radica en que son ellos, los miembros de las JUS, los que precisamente tienen como función no sólo proteger los derechos en salud sino la formación de nuevos miembros en las funciones señaladas, para el cual deberían tener tanto el conocimiento como la predisposición necesaria para tal fin.

Recomendamos, por tanto, que a nivel nacional y en todas las regiones del país y a todo nivel, inclusive local, se implementen las JUS con soporte técnico administrativo como estrategia de empoderamiento y enfoque social de la salud pública para que los propios usuarios formen parte de la protección y promoción de la salud.

\section{REFERENCIAS BIBLIOGRÁFICAS}

1. Muñoz F, López-Acuña D, Halverson P, Guerra de Macedo C, Hanna W, Larrieu M, et al. Las funciones esenciales de la salud pública: un tema emergente en las reformas del sector de la salud. Rev Panam Salud Publica. 2000;8(1-2): 126-134. DOI: http://dx.doi.org/10.1590/S102049892000000700017

2. Gutiérrez J, Hernández-Ávila M. Cobertura de protección en salud y perfil de la población sin protección en México, 2000-2012. Salud pública Méx. 2013; 55(Suppl 2):S83-S90.

3. Frenk J, de Ferranti D. Universal health coverage: good health, good economics. Lancet 2012;380(9845):862-864. DOI: 10.1016/S01406736(12)61341-5

4. Superintendencia Nacional de Salud [Internet] Reglamento de Organización y Funciones de la Superintendencia Nacional de Salud. Lima: SUSALUD; 2017 [Fecha de acceso: 18 de agosto de 2017]. Disponible en: http://portales.susalud. gob.pe/documents/11450/28161/ROF-2014 PDF/570fbc14-80a4-4a80-8549-65821390f13a

5. Velásquez A, Suarez D, Nepo-Linares E. Reforma del sector salud en el Perú: derecho, gobernanza, cobertura universal y respuesta contra riesgos sanitarios. Rev Peru Med Exp Salud Publica. 2016;33(3):546-555. DOI: http://dx.doi. org/10.17843/rpmesp.2016.333.2338

6. Arroyo J, Cáceres E, Chávez S, Rios M. Vínculos entre la Salud Pública y los Derechos Humanos. Lima: Consorcio de Investigación Económica y Social. Observatorio del Derecho a la Salud; 2004

7. Mezones-Holguín E, Díaz-Romero R, CastilloJayme J, Jeri-de-Pinho M, Benites-Zapata V, Marquez-Bobadilla Edith, et al. Promoción de los derechos en salud en Perú: una aproximación desde la perspectiva de acción de la Superintendencia Nacional de Salud. Rev Peru Med Exp Salud Publica. 2016;33(3):520-528. DOI: http://dx.doi. org/10.17843/rpmesp.2016.333.2302

8. Superintendencia Nacional de Salud [Internet]. Reglamento de la Junta de Usuarios de Servicios de Salud. Lima: SUSALUD; 2017 [Fecha de acceso: 18 de agosto de 2017]. Disponible en: http://portales.susalud.gob.pe/documents/11450/27670/Re glamento+de+la+Junta+de+Usuarios+de+los $+\mathrm{Se}$ rvicios+de+Salud.pdf/4a9fb7f9-16ae-4703-b8c7ffe67c79e06e

9. Altobelli L. Effectiveness in Primary Healthcare in Peru. In: E. Beracochea (Ed.) Improving Aid
Effectiveness in Global Health. New York: Springer Global Publishing. 2015: 161-170.

10. Ministerio de Salud [Internet]. Lima: MINSA; 2017 [Fecha de acceso: 18 de agosto de 2017]. Disponible en: http://www.minsa.gob.pe/cns/

11. Rebaza-Iparraguirre $H$, Vela-López M, VillegasOrtega J, Lozada-Urbano M, Munares-Garcia O. Desafios de la desconcentración y participación local en el marco de la protección de los derechos en salud: experiencia de la Intendencia Macrorregional Norte, SUSALUD. Rev Peru Med Exp Salud Publica. 2016;33(3):556-60. DOI:10.17843/ rpmesp.2016.333.2319

12. Gil-Quevedo W, Agurto-Távara E, Espinoza-Portilla E. Ciudadanos informados y empoderados: claves para el pleno ejercicio de los derechos en salud. Rev Peru Med Exp Salud Publica. 2017;34(2):3115. DOI: 10.17843/rpmesp.2017.342.2747

13. Superintendencia Nacional de Salud [Internet]. Encuesta Nacional de Satisfacción de Usuarios en Salud 2016. Lima: SUSALUD; 2017 [Fecha de acceso: 18 de agosto de 2017]. Disponible en: http://portales.susalud.gob.pe/web/portal/280

14. Delgado $M$, Vázquez María, Zapata $Y$, Hernán M. Participación social en salud: conceptos de usuarios, líderes comunitarios, gestores y formuladores de políticas en Colombia: Una mirada cualitativa. Rev. Esp. Salud Pública. 2005;79(6): 697-707.

15. Serrano C. Participación Social y Ciudadanía. Un debate del Chile contemporáneo. Chile; 1998.

16. Diario Oficial del Bicentenario El Peruano [Internet]. Normas Legales. Decreto Legislativo $N^{\circ} 1158$, Dispone Medidas destinadas al fortalecimiento y cambio de denominación de la Superintendencia Nacional de Aseguramiento en Salud. Disponible en: http://busquedas.elperuano.com.pe/ normaslegales/decreto-legislativo-que-disponemedidas-destinadas-al-fortal-decreto-legislativo-n-1158-1024507-3/

17. Delgado-Gallego M, Vásquez-Navarrete L. Barreras y oprtunidades para la participación social en salud en Colombia: percepciones de los actores principales. Rev. Salud pública. 2006;8(2):128-140.

18. Nigenda-López G, Juárez-Ramírez C, Ruiz-Larios J, Herrera C. Participación social y calidad en los servicios de salud: la experiencia del aval ciudadano en México. Rev. Saúde Pública. 2013;47(1):44-51. DOI: http://dx.doi.org/10.1590/ S0034-89102013000100007

19. Labra ME. Capital social y consejos de salud en Brasil: ¿un círculo virtuoso?. Cad. Saúde Pública. 2002;18(Suppl):S47-S55.

20. Méndez CA, Vanegas López JJ. La participación social en salud: el desafío de Chile. Rev Panam Salud Publica. 2010;27(2):144-8.

21. Jaime SF. Participación social en sistemas de salud fragmentados: ¿una relación virtuosa?. Rev. Gerenc. Polit. Salud. 2016;15(30):38-56. DOI: http:// dx.doi.org/10.11144/Javeriana.rgyps15-30.psss

22. Tanaka M. Participación popular en las políticas sociales Cómo y cuándo es democrática y eficiente, y por qué puede también ser lo contrario [primera edición]. Lima: Instituto de Estudios Peruanos (IEP) y Consorcio de Investigación Económico y Social (CIES). 2001.

23. Hevia FJ, Vergara-Lope S. ¿Cómo medir la participación? Creación, validación y aplicación del cuestionario de conductas de participación [Primera edición]. México D.F.: CIESAS-INDESOL. 2011. 